\title{
Un problema no es sólo un problema. Posiciones, hermenéuticas y políticas públicas relativas a la salud de las abejas
}

A problem is not just a problem. Positions, hermeneutics and public policies related to the bee health

\section{Guillermo Folguera}

Universidad de Buenos Aires-CONICET, Argentina

RESUMEN En diferentes lugares del mundo se discute acerca de la salud de las abejas y sus posibles causas. Argentina, uno de los principales productores de miel del mundo, inevitablemente ubica a esta problemática en un lugar central. En este trabajo indagaremos los modos de conceptualizar el problema y las hermenéuticas que le subyacen a diferentes agentes respecto a la salud de las abejas en Argentina. Con este fin, se realizará un análisis del vínculo entre posiciones y hermenéuticas de apicultores, Bayer, científicos de las áreas de la Toxicología y Biología del Comportamiento y funcionarios de diferentes áreas de SENASA, órgano de control del Estado argentino. Entre las conclusiones principales se reconoce: i) el acercamiento de las posiciones y hermenéuticas del Estado argentino y Bayer propio de un escenario neoliberal, ii) el modo en el que la Toxicología (área de mayor injerencia en la problemática) minimiza los riesgos ambientales respecto a la Biología del Comportamiento y iii) los numerosos procesos de simplificación que se reconocen en la toma de decisiones de la política pública en Argentina en los últimos años.

PALABRAS CLAVES Abejas; agroquímicos; hermenéutica; Estado.

ABSTRACT In different parts of the world, we discuss the health of bees and their possible causes. Argentina, one of the world's leading honey producers, inevitably owns this problem in a central place. In this work we will analyze the ways of conceptualizing the problem and the hermeneutics that underlie 
different agents regarding the bee health in Argentina. To this end, an analysis of the link between positions and hermeneutics of beekeepers, Bayer, scientists from the areas of Toxicology and Behavioral Biology and officials from different areas of SENASA, control body of the Argentine State will be carried out. Among the main conclusions it is recognized: i) Argentine State and Bayer present similar positions and hermeneutics suggesting a neoliberal scenario, ii) Toxicology (area of greatest interference in the problem) minimizes environmental risks with respect to Behavioral Biology and iii) numerous simplification processes are recognized in public policy decision making in Argentina in recent years.

KEY WORDS Bees; agrochemicals; hermeneutic; State.

\section{Introducción}

De manera creciente, diferentes aspectos asociados a temáticas ambientales y productivas están siendo reconocidas y discutidas. Una de ellas es la relativa a la salud de las abejas, que ha tenido alcances globales y que ha involucrado diferentes tipos de políticas públicas. Entre las razones de alarma, aparecen razones diversas: las ambientales, las productivas en relación con la práctica de la apicultura y las económicas directamente involucradas, dada la creciente dependencia de abejas para la polinización (Aizen, Garibaldi, Cunningham y Klein, 2008). Diversos autores acuerdan que se ha reconocido una disminución en la cantidad de abejas en Europa y América del Norte (ver por ejemplo Aizen y Harder, 2009; vanEngelsdorp et al., 2009). En muchos de esos casos, a la pérdida de abejas obreras adultas de las colonias y la falta de síntomas aparentes se le ha dado la denominación de "Sindrome de Despoblamiento de Colmenas" o SDC (CCD en inglés). Mientras algunos autores acotan el fenómeno de la CCD/SDC y de la muerte masiva de abejas a América del Norte y Europa, otros autores lo han planteado como una tendencia que afecta a todo el globo. De hecho, en el caso particular de Argentina se han registrado numerosas denuncias en los últimos años que marcan la disminución de la cantidad de colmenas (ver por ejemplo La Nacion, 8 de junio del 2018). Sin embargo, no hay acuerdos ni en cuanto al fenómeno ni a las causas que lo generan.

¿Qué involucra la percepción de un problema? ¿Qué elementos le subyacen? Cierto es que la conceptualización de un problema necesariamente está conectado con la persona y la comunidad que lo percibe, vive y enuncia. De este modo, no es posible darle un sentido único ni último a las necesidades que se reconocen ni a los fenómenos establecidos sin considerar además desde dónde es percibido. Se trata de una correlación entre comunidades y problemas. En cuanto a la pregunta por la salud de las abejas, muchas comunidades están directamente involucradas. Sea desde el cam- 
po científico, los agentes estatales de control, el sector científico o los apicultores, se multiplican agentes que no sólo perciben diferente la situación de la salud de la abeja sino que también se relacionan diferente con él.

A partir de todo lo mencionado, el objetivo general del presente trabajo es comprender y comparar los modos de conceptualizar y las hermenéuticas que tienen diferentes agentes en lo que respecta a la salud de las abejas en Argentina.

Con este marco general, el primer grupo de objetivos específicos está dirigido a la clarificación de las posiciones y las hermenéuticas que le subyacen a la posición de:

i) apicultores,

ii) responsables de la Dirección Nacional de Agroquímicos, Productos Veterinarios y Alimentos y el Programa Nacional de Sanidad Apícola del Servicio Nacional de Sanidad y Calidad Agroalimentaria (SENASA) , órgano de control del Estado argentino,

iii) comunidad científica en los campos de la Toxicología y la Biología del Comportamiento,

iv) la empresa Bayer en cuanto a su posición respecto a los insecticidas neonicotinoides y al programa Bee Care.

A su vez, el segundo grupo de objetivos específicos se dirige a la comparación entre cada una de dichas posiciones y hermenéuticas, y al nexo que presentan con las políticas públicas que se han implementado en Argentina en las últimas dos décadas con respecto a la salud de las abejas.

Con este fin el recorrido del trabajo es el siguiente. En la Sección 2 se realizará un análisis del vínculo entre las hermenéuticas, y los modos en que se posicionan diferentes agentes frente a problemáticas socio-ambientales. En la Sección 3 se presentará la metodología utilizada. En las siguientes secciones se caracterizarán las posiciones de diversos agentes y las hermenéuticas que le subyacen en cuanto a la salud de las abejas centrándonos en el caso particular de Argentina en los últimos años. A continuación, (Sección 4) se analizará el modo de conceptualizar el problema desde apicultores, tanto aquellos que organizan desde la Sociedad Argentina de Apicultores, como el modo en que lo evidencia una mujer que presenta a la apicultura como un eje fundamental de su vida en Río Cuarto (Córdoba). En la Sección 5 se examinará la posición de Bayer, empresa productora tanto de insecticidas de la familia de neonicotinoides, como de herbicidas también involucrados en la temática. En la Sección 6, se pone en foco la posición de los toxicólogos y los biólogos del comportamiento, para luego avanzar en la pregunta en relación de cómo se relacionan dichas publicaciones con la toma posterior de decisiones. En la Sección 7, se indagará la posición de funcionarios de diferentes áreas de SENASA. En la Sección 8 se establecerán diálogos entre las diferentes posiciones, analizando continuidades y discontinuidades entre las hermenéuticas involucradas. Finalmente, en la Sección 9 se señalarán las conclusiones principales. 


\section{Hermenéuticas y problemáticas}

¿Cómo indagar las percepciones diversas de un "mismo asunto"? Arranquemos pues comprendiendo un poco mejor qué entenderemos por hermenéutica. La hermenéutica, primero, como un proceso de interpretación. Esas aproximaciones adoptan nuevas direcciones en el siglo XIX con Schleiermacher y Dilthey. ¿Qué elementos deben considerarse en ese proceso? Al respecto, en la Enciclopedia Stanford (2019), se presentan siete preguntas fundamentales que la describen: quién es el autor, el asunto, por qué, cómo, cuándo, dónde, por qué medios fue escrito. Pero la forma más reciente de lo que involucra el proceso hermenéutico llegó con los enormes trabajos de Husserl, Heidegger, Gadamer, Foucault y Ricoeur, entre tantos otros.

A partir de entonces ya no se trata únicamente de un problema asociado al conocer, el análisis hermenéutico incorpora otras dimensiones. En esta dirección, uno de los aportes significativos que se han dado al respecto es de Paul Ricoeur. En su texto "Existencia y Hermenéutica" (1975), el autor señala que “...la hermenéutica pone en juego el problema general de la comprensión." (p. 8). Pero éste no se trata sólo "de un modo de conocimiento, sino [que es] un modo de ser" (p. 10). Interpretar involucra a los modos de existir.

Ahora bien, interpretación y existencia están vinculados, pero ¿cómo orientar una investigación en la que se reconocen una multiplicidad de agentes y comunidades? Cabe el recuerdo de Crisis de las Ciencias Europeas y la Fenomenología Trascendental de Husserl (1991 [1936]) en donde resulta fundamental el estudio del a priori de la correlación entre el objeto de la experiencia y modos de darse. Se trata del descubrimiento de la correlación en la cual toda variación en el modo de darse de un objeto responde a una manera diferente a la que el sujeto se orienta hacia él (Walton, 1993). En palabras de Walton (1993) esta correlación se convierte en un paralelismo entre el horizonte de capacidades potenciales del primero y el conglomerado de tipos empíricos que organizan los horizontes del segundo. El mundo de la vida así comprendido tiene un carácter a priori que es el que precisa ser reconocido e indagado. Otra vez, en palabras del propio Husserl, $\$ 38$ de la Crisis: “Todos nuestros temas teóricos y prácticos, también podemos decirlo de este modo, siempre residen en la normal unicidad del horizonte vital 'mundo'. El mundo es el campo universal en el que están dispuestos todos nuestros actos, experimentadores, cognoscitivos, activos" (Husserl, 1991 [1936], pp. 151-152). El mundo del científico, con las particularidades fundamentales del caso, es uno más entre los mundos. Por ello, las verdades en ciencia, se convierten en verdades dentro de su propio mundo circulante.

El proceso de indagación debe darse entonces en el horizonte de la conciencia histórica que debe ser considerado "desde dentro". En términos gadamerianos, se vinculan los prejuicios con la idea de un horizonte situacional inherente a toda experiencia comprensiva. Por supuesto que los prejuicios son el punto de partida, pero no 
permanecen estáticos sino que se modifican continuamente en el "movimiento" de la comprensión, y de este modo, representan la concreción de nuestra historicidad y el sello de nuestra finitud. Se trata de comprender nuestra pre-estructura de comprensión. No existe ni un sujeto puro ni un objeto puro. Todo objeto presupone un sujeto y todo sujeto presupone un objeto. En ese movimiento, entra en juego no sólo el conocimiento de lo conocido sino también de quién conoce.

De este modo, la pre-comprensión no sólo involucra percepciones y saberes, sino también acciones y deseos asociados a un sentido de vivir más amplio. A partir de lo señalado es que Wynne (2004) señala que en un debate no sólo se dan en el terreno de proposiciones verdaderas, sino también en terrenos hermenéuticos. Una hermenéutica que involucra el saber, el hacer y los presupuestos ontológicos. Así, tanto la consideración (o no) de cierto problema, el modo en el que el mismo se adopta como las propuestas que se le sugieren, involucran aspectos que configuran tanto las vías de conocimiento involucradas como su propia ontología. En particular, en este trabajo el foco estará en el modo en que se aborda el problema de la salud de las abejas.

\section{Metodología}

El trabajo de campo consistió en la realización de diversas entrevistas semiestructuradas a actores de diversa formación y pertenencia institucional con el fin de dar cuenta de las diferentes perspectivas sobre la problemática abordada. Asimismo, se complementó la investigación con el análisis de diferentes documentos institucionales escritos, sitios webs y artículos publicados en revistas de alcance internacional. Respecto del trabajo de campo se adoptó un abordaje microsocial y multisituado contemplando al "objeto de estudio como el entramado social donde se producen los intercambios y genera lo social, y donde el investigador está implicado" (Stagnaro, 2006, p. 97). Cabe señalar que se tuvo el resguardo ético de mantener la confidencialidad de todos/as los entrevistados/as.

Para el caso de la posición y hermenéutica de los apicultores se realizaron tres entrevistas en profundidad. Dos de ellas se efectuaron a pequeños apicultores de las afueras de la provincia de Buenos Aires. La entrevista restante se hizo a una apicultora de Río Cuarto, en la provincia de Córdoba. A su vez, con el fin de captar el punto de vista de los/as apicultores/as se complementó la indagación con material gráfico de la Sociedad Argentina de Apicultores (SADA).

Por otro lado, se retomó la perspectiva de los funcionarios estatales de este campo. En lo que refiere a la posición de SENASA se utilizaron para su análisis entrevistas de responsables de dos áreas de dicha institución: de la Dirección Nacional de Agroquímicos, Productos Veterinarios y Alimentos y del Programa Nacional de Sanidad Apícola. A su vez, se indagaron documentos oficiales de dicha institución. 
Asimismo, se complementó el abordaje incluyendo la perspectiva de científicos especialistas. Así, dentro de la posición de la comunidad científica se diferenció entre aquellos trabajos realizados desde el campo de la Toxicología y la propia de la Biología del Comportamiento. En cada caso se realizaron entrevistas a referentes de dichas áreas (tanto de Buenos Aires como de Santa Fe), a la vez que se sistematizaron los artículos de revistas especializadas de ambos campos de investigación publicadas en los últimos años.

Por último, para los fines de reconocer y estudiar la posición y la hermenéutica de Bayer se acudió a documentación institucional brindada por la propia empresa (a través de su sitio web) y trabajos científicos realizados y/o financiados por dicha empresa y publicados en revistas científicas.

\section{Apicultores}

"Las abejas disminuyen año a año en porcentajes variables, según la región" alerta la Sociedad Argentina de Apicultores (SADA, 2019). En términos generales, desde la percepción de los apicultores se reconoce que en los últimos años la salud de las abejas se ha visto fuertemente dañada. ¿Cuáles son las causas de dicho deterioro? En la página web de SADA se señala como las principales causas de este declive son las alteraciones de las condiciones ambientales en las que viven. En el hemisferio sur esta baja se debe principalmente a:

“1. El desarrollo del modelo agroindustrial impuesto en nuestro país basado en el monocultivo transgénico que determina la pérdida de biodiversidad y el hábitat de las abejas.

2. El uso intensivo de los agroquímicos que reduce su alimento, deteriora su capacidad de orientación y comunicación y finalmente las mata. Así, mueren aisladas, sin alimentos, ni conexión con su colonia” (SADA, 2019).

¿Cuál es el fenómeno que marca el deterioro de la salud de las abejas? Uno de ellos es el ya mencionado SDC/CCD, que se reconoce en general como el éxodo masivo de abejas de una determinada colmena. También se suele presentar como la muerte masiva de una colmena en un lugar dado. Sin embargo, no se trata de la única manifestación del deterioro de la salud en las voces de los apicultores. De hecho, los indicadores de stress por parte de aquellos apicultores trascienden en mucho la muerte y la vida de la abeja individual. Así, aparecen elementos asociados a su aspecto, al olor, a la agresividad de su comportamiento, a su nerviosismo, entre otros. En todos los casos, la mortalidad de las abejas individuales aparece como un factor más entre otros tantos involucrados. 
Conocer y existir están asociados. Por ello, resulta preciso volver a conceptos que parecen considerarse como evidentes y explicitar su sentido último: ¿qué es lo que entienden aquí por "abeja"? Un apicultor jerárquico en SADA señala que "el animal es el colectivo (...) ninguna abeja vive sola....se juegan la vida individualmente, pero no son individuales". En un mismo sentido, la apicultora de Río Cuarto señala que "La colmena es el animal (...) porque está formada por tres castas (...) Esa comunidad es la que es biológicamente activa (....) si falta algún integrante se genera un desequilibrio".

¿Cuáles son los causales de este fenómeno? Desde SADA se propone un escenario en donde son los efectos de los agroquímicos (que afectan directa e indirectamente a las abejas, tanto matándolas como sacándoles la comida) y del modelo agroindustrial, las que les impide obtener comida, a través de los desmontes y sacando de los territorios comunidades y apicultores. A su vez, realiza una analogía con el modo en que HIV afecta la salud de las personas y lo que se entiende como causa de muerte en tal caso. En el mismo sentido, la apicultora señala (2018): "Porque el agroquímico no es solamente un efecto de insecticida...el tema de los herbicidas te dejan sin flores con lo cual repercute en la nutrición y la nutrición repercute en la señal...está todo íntimamente relacionado..."

Por último, en el discurso de los apicultores, la disminución de las abejas necesariamente se asocia a una menor cantidad de trabajadores y comunidades que logran vivir de esta práctica. Uno podría señalar que se trata una consecuencia de lo primero, pero sin embargo se tratan de dos problemas asociados aunque diferentes. Así, como lo plantea otro de los miembros de SADA, se trata de "un momento grave de la apicultura" en la medida en que se pierden tanto abejas como apicultores. Desde esta perspectiva, la disminución de colmenas y de apicultores no son dos problemas: se trata de un mismo inconveniente con dos subproductos.

\section{Bayer y el cuidado de las abejas}

Otros de los actores claves fuertemente involucrados en la salud de las abejas pertenecen al sector empresarial. Se trata justamente de la empresa alemana Bayer, quien se relaciona con dicha problemática mediante, al menos, cuatro vías diferentes.

i) Por un lado, es una de las productoras principales del mundo en diferentes tipos de químicos que se los señala como posibles causales del daño sobre las abejas. En el caso de Argentina, tanto para los herbicidas como los insecticidas, su uso está muy extendido y fuertemente asociado al modelo agroindustrial (INTA 2015, KleffmannGroup, 2012). En los insecticidas de tipo neonicotinoides: "Desde 1991, Bayer ha producido el insecticida imidacloprid, que pertenece a los neonicotinoides, químicamente relacionado con la nicotina. Imidacloprid es uno de los insecticidas más utilizados en el mundo para el campo y los cultivos hortícolas. A menudo se utiliza como abono, especialmente para el maíz, el girasol y la colza. Bayer exporta más de 1.000 toneladas 
de Imidacloprid cada año a más de 120 países. Las ventas del año pasado fueron de 597 millones de euros. Dado que la protección de patentes para Imidacloprid venció en la mayoría de los países, Bayer sacó al mercado un neonicatinoide similar (denominado clotianidina) en el año 2003" (CBGnetwork.org 2019).

Frente a la moratoria y posterior suspensión de tres insecticidas neonicotinoides por parte de la Comunidad Europea, Bayer presenta una serie de evidencias acerca de la supuesta neutralidad de los neonicotinoides sobre la salud de las abejas. En su página oficial se referencia una publicación científica de autoría de Blacquière y colaboradores, publicado en la revista Ecotoxicology en el 2012. Bayer toma algunas de las conclusiones de dicho trabajo para concluir que: "Esos hallazgos están en línea con estudios a gran escala multifactoriales que fueron tomados en Estados Unidos, Austria, Bélgica, Canadá, Francia, Alemania y otros países. Ellos han mostrado que la salud pobre de las abejas está correlacionada con ácaros varroa, virus y otros muchos factores, pero no con el uso de insecticidas".

ii) Una segunda instancia de injerencia es el financiamiento de importantes investigaciones en diferentes partes del mundo. Una de ellas corresponde al trabajo de Woodcock et al. (2017) publicado en la revista Science en el que se obtuvieron diferentes resultados en los países analizados. Allí, se concluyen aspectos compatibles con la promoción de las denominadas Buenas Prácticas Agrícolas y en donde la falta de efectos homogéneos parece actuar en vías de permitir el uso de los insecticidas de tipo neonicotinoides (Woodcock et al., 2017). Al respecto, Science expande esta noticia, en la que Tjeerd Blacquière resume esta posición: "Nosotros aprendimos nuevamente: es complicado" (Science, 2017).

A su vez, el trabajo realizado por Fairbrother, Purdy, Anderson y Fellk (2014) publicado en la revista Environmental Toxicology and Chemistry también fue financiado por Bayer CropScience Ag Research Division. Aquí señalan: "The available data indicate that there may be effects to individual honeybees housed under laboratory conditions and exposed to unrealistically high concentrations of the insecticides. However, under field conditions and exposure levels, similar effects on honeybee colonies have not been documented. It is not reasonable, therefore, to conclude that crop-applied pesticides in general, or neonicotinoids in particular, are a major risk factor for honeybee colonies, given the current approved uses and beekeeping practices" (p. 729). Según el trabajo los resultados experimentales no pueden usarse en políticas públicas. Esto será retomado en la Sección 7.

iii) Al comprar a la empresa Monsanto, la empresa Bayer ha pasado a ser propietaria del herbicida glifosato, que en el caso de Argentina es el químico más utilizado en los territorios desde hace más de dos décadas. Los agroquímicos no son una novedad para Bayer. De hecho, para concretar ese negocio Bayer le vendió a BASF su herbicida basado en glufosinato de amonio (Agrovoz, 2017). La salud de las abejas, tal como 
veíamos desde la percepción de los apicultores, está fuertemente asociada a los herbicidas por incidir en la disponibilidad de alimento de las abejas. A su vez, tal como veremos más adelante, diferentes estudios han sugerido que el glifosato puede actuar negativamente de otros modos.

¿Qué señala Bayer respecto al glifosato? En su página web señala que "glyphosatebased herbicides will continue to play a key role in helping agriculture meet pressing environmental and food security challenges as the global population grows by an expected two billion people through 2050" (Bayer, 2019). Y luego señala: "Glyphosate is one of the most studied herbicides in the world - and, like all crop protection products, it is subject to rigorous testing and oversight by regulatory authorities. There is an extensive body of research on glyphosate and glyphosate-based herbicides, including more than 800 scientific studies and reviews submitted to U.S., European and other regulators in connection with the registration process, that confirm that glyphosate and our glyphosate-based formulated products can be used safely and are not carcinogenic. The EFSA, the U.S. EPA and other regulatory authorities around the world have comprehensively and routinely reviewed glyphosate and glyphosatebased herbicides for more than 40 years and their conclusions consistently support the safety of glyphosate and glyphosate-based herbicides when used as directed." (Bayer, 2019).

iv) Por último, Bayer presenta un proyecto bee-friendly. En su promoción y descripción, nuevamente se intensifica la idea de buenas prácticas: "cuyos objetivos son desarrollar soluciones innovadoras para mejorar la salud de las abejas, para promover activamente el uso responsable de nuestros productos, así como fomentar el conocimiento y la experiencia con todos los actores e instituciones (apicultores, agricultores, instituciones de investigación, organizaciones no gubernamentales), así como con los responsables políticos y las autoridades reguladoras" (Bayer-Beecare, 2019). En su página general, se señala al respecto que:

"Records suggest that the number of managed honey bee colonies has declined in certain regions, such as in North America and Europe, but has increased in other areas, such as in Asia, Africa and South America. Within regions, the number of colonies can vary considerably: Researchers found declines in colony numbers in central European countries but increases in Mediterranean countries. Even in the areas that have seen declines over the past 50 years, there is recent evidence showing that colony numbers have either stabilized or are increasing again." (Bayer-Beecare, 2019). Se niega el problema global y se aclara que en nuestra región el número ha incrementado. Ya veremos su consonancia con el discurso de los órganos de controles locales.

Creencia, mitos y datos. Bayer alerta "Don't Believe Everything You Hear!". Más adelante volveremos a este asunto pero en un contexto ligeramente diferente. También allí se señala: "Nearly all experts agree that honey bee health is affected by many 
factors. These include adverse weather, inadequate availability of forage, parasites, diseases, genetic problems and inappropriate hive or crop management practices. $\mathrm{Re}-$ moving one factor - such as pesticides - does not mean that bee health will suddenly improve" (Bayer-Beecare, 2019, p. 20).

Y luego, en lo referido a neonicotinoides se indica que:

- "Scientists evaluated 61 factors and found none that stood out as the primary cause of CCD.3

- Documented incidences of CCD are rare. In 2018, the bee researcher who coined the term "CCD" reported that he had not seen a credible case of its reoccurrence in five years.

- Mentions of CCD are still seen in print and social media but have been in consistent decline since reaching a peak of more than 8,400 articles in 2013" (BayerBeecare, 2019, p. 36).

Como podemos ver, la posición de Bayer presenta una serie de características distintivas. Mientras se niega el carácter global del CCD/SDC, busca mostrar los efectos de interacción y la multiplicidad de factores que lo afectan dando un lugar menor a la injerencia química y mayor a las buenas prácticas, se sostiene fuertemente en lo científico. Por todo ello resulta imperioso comprender cómo se gesta el discurso profesional, cuán representativo es lo retomado por Bayer y qué elementos hermenéuticos se reconocen en relación con el modo de conceptualizar el problema en cuestión.

\section{Abordajes científicos}

\section{a. Toxicología}

Otro de los ámbitos desde los que se abordó el problema de la salud de las abejas fue el científico. En particular, fue la Toxicología una de las subdisciplinas centrales que indagaron en las últimas décadas acerca de la salud de las abejas. Cabe señalar que se trata de un campo que reúne a su vez aportes de áreas diferentes como la toxicología ambiental, la ecotoxicología y la genotoxicidad (ver por ejemplo Frickel, 2004; Newman y Unger, 2003; Wright y Welbourn, 2002). La pregunta central, a partir del tipo de abordaje que hemos elegido es cómo se ha configurado el problema desde este meta-campo. Cierto es que como cualquier otra área del conocimiento, al abordarlo se apropia de sentidos, haciendo suyo no sólo al problema, sino también a posibles vías de intervención sobre él. Ahora bien, ¿qué tipo de transformaciones han operado para el caso de la Toxicología?

La estrategia metodológica que predomina en los estudios realizados desde la Toxicología está dada en condiciones controladas de experimentación. Y es en el análisis de esos estudios en donde se comprende la transformación inicial que realiza dicho campo. En particular, se suele considerar en los estudios toxicológicos el de- 
nominado NOAEL, que es entendido como la máxima concentración o nivel de una sustancia en las que no se encuentran alteraciones adversas detectables bajo condiciones definidas de exposición. Otro de los parámetros que suele utilizarse es la dosis letal 50 (LD50), entendido como la cantidad de un material que provoca la muerte del $50 \%$ (una mitad) de un grupo de animales de prueba. Por ello, el LD50 es una forma de medir el envenenamiento potencial a corto plazo (toxicidad aguda) de un material.

En dicho abordaje, el primer modo en que se expresa el horizonte de sentido aparece en la relación entre los fenómenos a explicar y las causas propuestas. De este modo, pueden verse modificaciones en los fenómenos que buscan ser explicados. $\mathrm{O}$ dicho de otro modo: ¿se están buscando las causas que explican qué fenómenos? Tal como plantea la ecotoxicóloga Celina Junges durante la entrevista, la muerte masiva de abejas y el fenómeno de SDC, son fenómenos diferentes que no necesariamente responden a causas similares.

Sin embargo, la búsqueda de causas globales es una de las características propias de las ciencias naturales. En este sentido, una parte importante de los estudios de Toxicología que han abordado fenómeno de la salud de las abejas se han centrado en el análisis de los efectos de los insecticidas de tipo neonicotinoides, presentes ambientalmente a escala global y también en nuestros países. En el modo de hacer operativo el abordaje desde la Toxicología, se registra el inevitable desplazamiento de los sentido. Ahora bien, ¿cuáles son las coordenadas de dicha apropiación por la pregunta acerca de la salud de las abejas? En una primera instancia se reconoce una primera transformación en la que el problema de la salud de las abejas se convierte en cuáles son los efectos de los neonicotinoides (ver por ejemplo: Lundin, Rundlöf, Smith, Fries y Bommarco, 2015). Ahora bien, ¿qué otras transformaciones recibe el problema tal como es abordado el problema de la salud de las abejas? ¿Qué otras determinaciones recibe desde la propia Toxicología? Veamos cinco de ellas:

i) En la mayor parte de los casos, el efecto de cada uno de los químicos es estudiado de manera singular, sin evaluar sus interacciones. $\mathrm{Al}$ respecto, Blacquiere et al. (2012) señalan: "Only one study is available on the toxicity of neonicotinoids in mixtures to pollinators. Iwasa et al. (2004) found that addition of piperonyl butoxide and the fungicides triflumizole and propiconazole increased the acute toxicity (24-h LD 50, topical application) of acetamiprid and thiacloprid to honey bees (A. mellifera) by factors of 6.0, 244 and 105, and 154, 1141 and 559, respectively, but had little effect on the toxicity of imidacloprid (1.5-1.9 times more toxic). The toxicity of acetamiprid was 6.3-84 times increased by the fungicides triadimefon, epoxiconazole and Uniconazole-P. (...) The data do, however, not allow for a quantitative risk analysis of possible mixture exposure." (p. 986). Por supuesto que hay excepciones en los que las interacciones (en general de a pares) sí se consideran e indagan (ver por ejemplo: Johnson, Pollock y Berenbaum 2009; Pilling y Jepson 1993; Thompson y Selwyn 
2003; Zhu, Yao, Adamczyk y Luttrell, 2017). Sin embargo, por el tipo de abordaje en la que sólo excepcionalmente se consideran y evalúan interacciones de químicos esta característica hace de la determinación una clave para comprender las alteraciones y significaciones asociadas.

ii) En el campo de la Toxicología prevalecen los estudios sobre efectos agudos y letales. En el ya mencionado paper de Blacquiere et al. (2012) se reconoce este sesgo disciplinar: "During the last years a loss of overwintering bee colonies was noticed. Although identification of the causes of this disappearance is difficult, it was argued that reduced bee health might be initially caused by the chronic exposure to pesticides. So far only two studies have been conducted in this context for neonicotinoids. (...) In conclusion, these studies demonstrated no long-term effects on honeybee colonies of environmentally relevant concentrations." (p. 986). Del mismo modo, en palabras de Lundin et al. (2015) "Furthermore, chronic toxicity for bees at time points beyond a standard 10 days test time was rarely considered, even though neonicotinoids might cause delayed and time-cumulative toxicity" (p. 12). Al igual que en el caso de la interacción entre químicos, las pocas evidencias parecen sugerir que los estudios crónicos pueden ser significativos. Por ejemplo, la investigadora española Concepción Ornosa, directora del grupo de Biología y Biodiversidad de Artrópodos de la Universidad Complutense de Madrid señala "que los neonicotinoides no parecen afectar negativamente al abejorro común y a la abeja solitaria el primer año del tratamiento, pero sí el año siguiente (...) [y esto] pone de manifiesto la importancia de la persistencia de los residuos de neonicotinoides'”' (https://elpais.com/elpais/2017/06/29/ciencia/1498753026_858601.html). Por supuesto que hay excepciones las cuales serán justamente recuperadas en la Sección 7 (ver por ejemplo Suchail, Guez y Belzunces 2001; Williamson, Willis y Wright 2014).

iii) Propio de los estudios experimentales es también el centro de individuos aislados aún sociales, y la dificultad por indagar a la colmena como un todo. En palabras de Lundin et al. (2015) al analizar los trabajos realizados desde la toxicología para indagar los efectos de los neonicotinoides sobre las abejas se reconoce que: "Most studies measured the effects of neonicotinoids on bees at the individual level ( $\mathrm{n}=109$ studies), followed by the colony level $(n=60)$ and the sub-individual level $(n=48)$. None of the studies investigated effects on the population level according to our definitions. Studies that measure the effects on the individual were most common. (...) Nevertheless, the colony level is of primary interest in managed honey bees, because reduced colony performance and higher rates of colony loss directly affect beekeepers." (p. 12). Evidentemente, la transformación se apoya en limitaciones y características teórico-metodológicas. Al respecto "One reason for the relative paucity of colony level studies may be methodological difficulties of performing replicated studies on honey bees, which have large and variable colony sizes and strengths. Future research should therefore try to overcome these difficulties, for example by increasing 
sample sizes (see e.g. [98]) and employing increased levels of standardization among colonies in order to determine how neonicotinoids affect honey bee colony performance." Las restricciones metodológicas que en efecto se trazan, dejan sin embargo preguntas respecto al modo en que serán consideradas para las decisiones de políticas públicas.

iv) Aunque con excepciones, la mayor parte de las investigaciones en el área de la Toxicología se centran en estudios sobre los organismos adultos y sólo excepcionalmente en los estadios larvales (ver como excepción Tan et al., 2015). Por cierto, este sesgo es una constante en una gran parte de las investigaciones asociadas a los seres vivos. Sin embargo, es claro que frente a la búsqueda de reconocimiento de potenciales riesgos, los estadios juveniles suelen ser notablemente más susceptibles. En palabras de Lundin et al. (2015): "Most studies measured the effects on the adult life stage whereas the effects on brood, larval or pupal stages received much less attention. Exposure to neonicotinoids in early life stages of bees can have both direct lethal effects and delayed sublethal effects on the adults." (p. 12). Sin embargo, los análisis en estadios más juveniles no sólo pueden diferir significativamente en la sensibilidad de los organismos hacia los químicos. Al tratarse de un insecto holometábolo, los estadios de huevos, larvas, pupas y adultos pueden mostrar no sólo diferencias cuantitativas, sino que podrían evidenciar verdaderos desacoples y novedades cualitativas.

v) Por último, prácticamente todas las investigaciones estudian los efectos dentro de una misma generación. Como suele pasar en otros campos disciplinares, raramente se estudia el modo en el que incide a través de las generaciones. Sin embargo, estos tipos de estudios no sólo son difíciles en cuanto a su implementación en términos de políticas públicas, sino que en muchas ocasiones resultan complejos en los términos de las investigaciones en juego.

Comprendidos como un todo, los cinco aspectos señalados representan parte del desplazamiento de sentido propio dada por la mayor parte de las investigaciones del campo general de la Toxicología. Así, frente a la pregunta por los efectos de los neonicotinoides, la respuesta está dada por la salud de las abejas individuales, intrageneracionalmente, adultas y con efectos agudos. Por supuesto, esta transformación de la problemática presenta nexos con un modo general de pensar este tipo de problemática. En palabras de Kleinman y Suryanarayanan (2013) se señala que: “...esta forma epistémica, cual viene a ser dominante en las investigaciones toxicológicas ambientales sobre las abejas, fue estructurada para medir factores individuales y sus roles causales. (...) Esto no podría seriamente o sistemáticamente considerar interacciones plausibles con factores ambientales tales como otros pesticidas y patógenos. Entonces, esta forma epistémica fue estructurada de tal modo que no podría considerar un grupo complejo de factores interactuantes que plausiblemente lleven a efectos lentos, progresivos sobre múltiples generaciones en el ciclo de vida de las colmenas" (pp. 497-498). 


\section{b. Biología del Comportamiento y otros campos afines}

Otro de los campos científicos que ha abordado la temática de la salud de las abejas es el de la Biología del Comportamiento. Tal como en el caso de la Toxicología, este campo disciplinar también presenta en su interior una diversidad muy significativa de aproximaciones. Así, en la actualidad pueden reconocerse enfoques de tipo genético, neurobiológico, ontogenético, ecológico y evolutivo (Pallitto, 2018). Niko Tinbergen señaló en la década de 1960 cuatro grandes preguntas que abordaba por entonces la biología del comportamiento, siendo una de las posiciones más aceptadas por entonces (Bateson, 2012, Bateson y Laland, 2013). Junto con la diversidad de marcos teóricos, la proliferación de subdisciplinas generó una diversidad de aproximaciones metodológicas que hicieron de este campo un escenario híbrido, y de gran riqueza y complejidad.

Ahora bien, ¿cómo se manifiesta esta aproximación en cuanto a la forma de conceptualizar el problema de la salud de las abejas? En principio surgen dos elementos $a$ priori: mientras la Toxicología y sus diferentes subcampos asumen el problema desde la perspectiva del químico en cuestión, la Biología del Comportamiento lo hace desde el ser vivo y su comportamiento. Este cambio redunda en diferentes aspectos y características que son evaluadas. Del mismo modo, la gran expansión y riqueza metodológica genera que las formas de acercarse a la temática también presentan diferencias significativas al seno del campo.

Sin la búsqueda de ser exhaustivos, es claro cómo los estudios realizados desde la Biología del Comportamiento presentan inclusiones más frecuentes en cuanto a efectos crónicos y subletales (ver por ejemplo: Herbert, Vázquez, Arenas y Farina, 2014 respecto a las respuestas a determinados estímulos de olor y de memoria, Balbuena et al., 2015 y Fischer et al., 2014, Tosi, Burgio y Nieh, 2017, en lo referido a la navegación aérea de las abejas; Tsvetkov et al., 2017). A su vez, se reconocen estudios en adultos jóvenes (Goñalons y Farina 2015) y en las colmenas (Goñalons y Farina, 2018; Vázquez, Ilina, Pagano, Zavala y Farina, 2018; Whitehorn, O'Connor, Wackers y Goulson, 2012), en la medida en que es reconocido en mayor grado el efecto de un insecto holometábolo y social. También es muy interesante la aparición de estudios relativo a los efectos directos de un herbicida como el glifosato sea de manera aislada (Balbuena et al., 2015; Herbert et al., 2014), o en interacción entre químicos (Goñalons y Farina, 2018). Por supuesto, que al igual que en el caso de la Toxicología, aparecen numerosos contraejemplos, por lo que la caracterización de un campo del saber es necesariamente temerario. Sin embargo, a nuestros fines basta reconocer las diferencias entre ambos campos no sólo hacia adentro de los propios abordajes profesionales, sino también tal como veremos, en cuanto a la toma de políticas públicas. 


\section{Estados, políticas públicas y la salud de las abejas}

\section{a. Estados europeos y los neonicotinoides}

En 2013 la Comunidad Económica Europea decidió una moratoria respecto a los agrotóxicos de tipo neonicotenoides. En el 2018, siguiendo los delineamientos de la Autoridad Europea de Seguridad Alimentaria (EFSA) los Estados europeos concluyeron que tres insecticidas -imidacloprid y clotianidina, fabricados por Bayer, y tiametoxam, de Syngenta - “"'en general” representan "un riesgo para las abejas silvestres [abejorro común y abeja solitaria] y las abejas melíferas". Estos pesticidas, de uso frecuente en todo el mundo en cultivos de maíz, girasol, colza y algodón, están sometidos a restricciones en la UE desde 2013, en aplicación del principio de precaución"”' (El País, 2018).

De este modo, los Estados de la Comunidad Económica Europea decidieron su prohibición en sintonía con el dictamen de la EFSA. La explicación de dicha prohibición es que la "EFSA compared the expected levels of neonicotinoid pesticides to which bees are likely to be exposed in the environment to those that cause effects to bees. Whenever the estimation of the environmental contamination was higher than the levels considered safe for bees, a high risk was concluded. For all the outdoor uses of these substances, there was at least one aspect of the assessment indicating a high risk, leading to the conclusion that overall these neonicotinoids represent a risk to bees. (...) In 2015 EFSA carried out an open call for data from studies, research and monitoring activities relevant for the uses under consideration. EFSA also considered the data available from the systematic literature review carried out for the previous assessments in 2013. Furthermore, an update of this systematic review was performed in June 2016, to collect all published scientific literature relevant for the current evaluation. Data was received from academia, beekeeper associations, chemical companies, farmers' associations, NGOs and national authorities. More than 1,500 studies were considered by EFSA prior to beginning the assessments." (EFSA, 2018).

\section{b. Al sur de Sudamérica}

¿Qué actitud ha tomado el Estado argentino en este debate? Respecto a la pregunta general acerca de la salud de las abejas, en una primera instancia se reconoce el mismo desplazamiento de sentido coincidente con los Estados europeos y con lo señalado Bayer. Sin embargo, las conclusiones obtenidas fueron al respecto: no sólo no hubo prohibiciones al respecto, sino que el SENASA autorizó 6 registros de clotianidina (todos de Bayer) y 206 de imidacloprid (12 de Bayer). En el 2013 respecto a la decisión europea acerca de los neonicotinoides SENASA se expidió negando tanto sus considerandos como sus conclusiones: 
"Atento a la preocupación manifestada por productores apícolas, y toda la cadena productiva asociada, respecto del impacto negativo de la aplicación de productos fitosanitarios cuyos principios activos corresponden al grupo de los neonicotinoides, es necesario hacer algunas consideraciones sobre la iniciativa de la Unión Europea de restringir los principios activos de este grupo, Clotianidina, Imidacloprid y Tiametoxam.

- La restricción se basa en una revisión de la Autoridad de Seguridad Alimentaria Europea (EFSA), que ha sido ampliamente criticada por sus reconocidas debilidades técnico-científicas. Esto se debe a que la evidencia científica no demuestra una correlación entre la salud de las abejas y el uso de neonicotinoides.

- Los estudios realizados son de carácter parcial y no consideran la multiplicidad de factores que pueden ser causantes de afectar la salud de las abejas. En los Estados Unidos, el Departamento de Agricultura (USDA) y la Agencia de Protección Ambiental (EPA) ha publicado que la disminución de abejas melíferas obedece a un conjunto de causas variadas.

- La Ciencia claramente ha demostrado que las abejas y otros polinizadores pueden coexistir de manera segura con las tecnologías agrícolas modernas, tal como es el caso de los neonicotinoides.

- Los insecticidas neonicotinoides han sido usados de manera segura alrededor del mundo durante varios años. Restringir su uso no es una alternativa que garantice o fomente la salud de las abejas, por el contrario podría implicar un retroceso en la agricultura moderna." (PortalApícola, 2013).

Dicha declaración fue una de los pocos pronunciamientos oficiales respecto a la salud de las abejas y potenciales causas. Por ello, se realizaron tres entrevistas a integrantes de SENASA. La primera fue realizada a un agente de alto rango del Programa Nacional de Sanidad Apícola. Respecto al interrogante acerca de si hay una crisis de la salud de las abejas, el agente en principio señaló que el CCD es más una problemática "del Hemisferio Norte (...). En el Hemisferio Sur no se da de forma preocupante (...) no se da en forma alarmante, masiva." A la vez, agregó que "una cosa son las creencias y otros los datos". Sin embargo, SENASA no parece tener "datos confiables" ya que tal como planteó el tercer entrevistado de dicho organismo "los apicultores no informan nada porque le tienen miedo a AFIP y a SENASA". A su vez, el agente de alto rango entró directo en la discusión acerca de los neonicotinoides señalando que nunca detectaron neonicotinoides aunque "no sabés en definitiva qué es lo que está pasando en ese cóctel de sustancias". Esta posición, sin embargo, mostró fluctuaciones a través de la entrevista sugiriendo incluso tres tipos de causas diferentes: una dada por la pérdida de biodiversidad, un manejo sanitario no adecuado y, finalmente, la presencia de plaguicidas en el ambiente. Incluso, respecto a los propios datos recolectados por 
SENASA señala que no son muy representativos aunque "estamos muy lejos de la alarma”. En sus palabras, dicho comunicado fue elaborado por la Dirección de Agroquímicos de SENASA aunque firmado de manera general. Luego agregó que "Desde el Programa hicimos un informe no compartiendo sus criterios y respondiendo cada uno de sus puntos". Sin embargo, ante el pedido nos señaló que no era posible compartirlo.

La segunda entrevista fue realizada a un agente de la Dirección de Agroquímicos de SENASA quien confirmó que el comunicado en efecto había sido generado por su Dirección. La normativa que rige la aprobación de los químicos es de la década de 1990 (Resolución 350/99) con influencia de la FAO y señala que tanto los estudios para aprobar los agroquímicos como aquellos en instancias posteriores están a cargo de las empresas. Esto que rige para agroquímicos, no se da en otros casos tales como el análisis de aftosa o de sarna ovina en los que SENASA interviene de manera directa ¿Por qué confiar en las empresas en el caso de agroquímicos? La respuesta se dirigió a dos aspectos diferentes. En primera instancia, hay una profunda confianza en los protocolos y en las buenas prácticas agrícolas, productivas y apícolas, tal como se veía en el caso del discurso de Bayer. A su vez, ese explicitó una confianza inherente en la ética empresarial como un aspecto vertebral de todo el proceso de evaluación. Cabe señalar también que en ningún caso interviene el Ministerio de Salud. A su vez, los vínculos con los académicos como con actores no profesionales apareció como periférico y circunstancial. Otro aspecto a mencionar de la entrevista con el agente de la Dirección de Agroquímicos, fue la explicitación de que SENASA no se rige con el principio de precaución sino con uno alternativo que sopesa beneficios/daños: se aprueba el agroquímico en cuestión cuando los prometidos beneficios superan a los potenciales daños.

En todos los agentes entrevistados, los productos químicos no fueron objeto de cuestionamiento ni de potencial efecto negativo sobre la salud de las abejas. En este sentido, cabe recordar cómo el Ministro de Agroindustria Luis Miguel Etchevehere enfatizó que el modelo agroindustrial no podría tocarse, por lo que se continuará con los agroquímicos y el modelo tecnológico que conforma con los OGMs: ““¿Cómo piensan convivir con eso? Porque el modelo no va a cambiar", disparó el ministro en pleno intercambio" (Infocampo.com.ar, 2018).

\section{Hermenéuticas, abejas y la configuración de las políticas públicas}

Los cuatro grupos de posiciones junto con sus hermenéuticas, en una primera instancia nos muestran un mapa fuertemente diverso. Sin embargo, tal diversidad no parece expresarse en las discusiones y políticas públicas acerca de la salud de las abejas que se han dado en Argentina. Más aún, frente la discusión que se ha dado en algunos lugares del globo, siquiera parece asumirse que puede estarse dando un problema serio 
en Argentina. En ese sentido, los diferentes actores de SENASA coincidieron en negar que se trate de un momento de crisis de las abejas, al igual que Bayer cuando señala que América del Sur ha incluso mostrado crecimiento en el número de poblaciones de las abejas a diferencia del Hemisferio Norte. Sin embargo, esta percepción no es coincidente con los apicultores locales, quienes no dudan mayormente en cuanto al fenómeno.

Otro aspecto a destacar es el modo en que intervienen la mayoría de los profesionales que forman parte de la comunidad científica. Nuevamente, la pregunta general sufre un desplazamiento para interrogar si los insecticidas neonicotinoides son sus principales causas. Inevitablemente, la estrategia por hacer operativa la pregunta genera necesarias transformaciones. Así, junto con esta primera transformación, se reconocen nuevas determinaciones propias de los campos del saber que los indagan que van desde los estudios de los efectos letales o subletales, el impacto sobre la abeja individual o sobre la colmena, efectos agudos o crónicos, efectos en adultos o en otros estadios, efectos intra o intergenacionales, etc. No se trata de meras cuestiones técnicas, lo que subyace a ellos, tiene mucho que ver con el percibir y el hacer. Siguiendo a Wynne, dichas posiciones diferentes no radican estrictamente a un contrapunto proposicional, sino más bien a horizontes de sentido, que se vinculan con saberes y con prácticas diferentes.

Esta multiplicidad de determinaciones correlaciona con una diversidad de aproximaciones tanto entre campos del saber (representados aquí por la Toxicología y la Biología del Comportamiento) como dentro de cada una de las subdisciplinas. Sin embargo, la diversidad no es recuperada ni por parte del sector empresarial ni por los integrantes de los órganos de control del Estado.

Hacia dentro de la comunidad científica también es frecuente encontrar algunas coincidencias, aún en investigaciones que no son directamente financiadas por las empresas involucradas. Por ejemplo, Fairbrother et al. (2014) señalan: "Laboratory testing allows for greater control over environmental conditions and reduces the impact of extraneous factors. However, as discussed in more detail in the Field and semifield tests section below, caution is needed when attempting to extrapolate the results of laboratory studies to the colony level" (p. 724). Del mismo modo: "Bee colonies have emergent properties that make it very difficult to extrapolate colony-level exposures from honey and bee bread and colony-level responses to field applications of chemicals." (p. 729). También Blacquière et al. (2012) reconocen dicha discontinuidad al señalar que: "Many lethal and sublethal effects of neonicotinoid insecticides on bees have been described in laboratory studies, however, no effects were observed in field studies with field-realistic dosages." (p. 988). Del mismo modo, Lundin et al. (2015): "Based on our systematic literature review we conclude that despite considerable research efforts, there are still significant knowledge gaps concerning the im- 
pacts of neonicotinoids on bees. We found that studies were not representative of the diverse and global use of neonicotinoids that are applied in a multitude of insect pollinated crops and non-crop plants that are often visited by multiple bee species." (p. 13). O bien que estamos frente a una multiplicidad de causas en donde los tóxicos ocupan lugares menores: Fairbrother et al. (2014). While beekeepers may have difficulty diagnosing a new phenomenon such as colony collapse disorder, they are familiar with other causes of colony loss; and pesticides ranked 8th on the list of possible causes of colony loss in the USDA survey (p. 729).

¿Qué posicionamientos y hermenéuticas presenta el Estado argentino? En principio, el CCD/SDC siquiera es aceptada en el Cono Sur, por lo que los neonicotinoides no son siquiera evaluados en cuanto a sus efectos. A su vez, los miembros de SENASA a través de las figuras de "datos y creencias", presentan sus propios registros como confiables (a pesar de los problemas que ellos mismos reconocen) y que sólo hay falsas percepciones por parte de los apicultores.

Hay otros dos aspectos presentes en la hermenéutica del Estado. Por un lado, un privilegio de los profesionales. La pluma de Illich (2015) en la década de 1970' ya alertaba: "se recordará la Edad de las Profesiones como aquel tiempo en que la política entraba en descomposición cuando los ciudadanos, guiados por profesores, confiaban a tecnócratas el poder de legislar sobre sus necesidades, la autoridad de decidir quiénes necesitaban qué cosa y el monopolio de los medios que satisfacían esas necesidades" (p. 499). De todos modos, este privilegio de los profesionales no sólo actúa considerando al posicionamiento de los apicultores como meras "creencias", sino que retoma sólo algunos de los trabajos realizados en uno de los dominios disciplinares, negando la diversidad en la que está involucrada como el desplazamiento de sentido involucrado respecto a la pregunta más general.

El otro aspecto se dirige al modo en que al suspender el principio de precaución y abonar por un análisis de sus "beneficios" y "costos", el fin de acumulación económica es el prioritario. Esto reproduce la lógica analizada en Bayer, ahora dada desde el propio Estado argentino. Surge inevitable entonces para comprender los alcances de ello la racionalidad que gobierna a los Estados de corte empresarial-neoliberal. Ahora bien, ¿qué características centrales tiene el neoliberalismo? Brown (2017) resume: "La manera más común de entender el neoliberalismo es como el ensamble de políticas económicas que coinciden en su principio original de firmar libres mercados. Éstos incluyen la desregulación de las industrias y de los flujos de capital; la reducción radical de las provisiones del Estado de bienestar y de sus protecciones para quienes son vulnerables; la privatización y subcontratación de bienes públicos, que van desde la educación, los parques, los servicios postales, las carreteras y la previsión social hasta las cárceles y los ejércitos; el reemplazo de esquemas hereditarios y de arancel progresivos por regresivos; el fin de la redistribución de la riqueza como una política 
económica o sociopolítica; la conversión de cada necesidad o deseo humano en una empresa rentable, desde la preparación para ser admitido en universidades hasta los trasplantes de órganos, desde las adopciones de bebés hasta los derechos de contaminación, desde evitar colas hasta asegurar un espacio cómodo en un avión y, más recientemente, la financiarización de todo y el creciente dominio del capital financiero sobre el capital productivo en la dinámica de la economía y la vida cotidiana" (Brown, 2017, p. 30). Así, "se da prioridad a la productividad sobre el producto, a emprender sobre el consumo o la satisfacción.” (Brown, 2017, p. 83). Estas dos características hermenéuticas, muestra en Argentina formas precisas.

El caso particular aquí indagado y las formas en que tomó, refuerza aquello señalado por Böschen, Kastenhofer, Rust y Soentgen (2010) en que se trata de una cultura científica orientada al control/paradigma químico. Por cierto hay algunas diversidades, algunos acuerdos parciales, algunos acuerdos profundos. Hermenéuticas de los Estados y un rol del Estado argentino que pese a presentarse con cierta diversidad interna que no se ha expresado en cuanto a sus prácticas. Pero no sólo se trata de un privilegio de lo químico. También es la estrategia para hacer proliferar negocios, incluso en lo que respecta a la propia polinización (por ejemplo hoy mas del 65\% de las colonias en Estados Unidos están siendo usadas para polinización (Fairbrother et al., 2014, ver también https://ecocolmena.com/servicios-de-ecopolinizacion/). El desplazamiento de sentido de la pregunta por la salud de las abejas, el modo en que es tratado el beneficio económico respecto a potenciales daños ambientales y sociales, y las apoyaturas en la propia ética empresarial sin análisis propios en el caso de los agroquímicos, son algunos de los elementos distintivos que se expresan al respecto en la hermenéutica del Estado argentino.

\section{Conclusiones: pluralidad de hermenéuticas, políticas públicas y ontologías mo- nistas}

La pregunta original se desdobló en dos interrogantes derivados. La primera es si el fenómeno que se ha observado en el Hemisferio Norte en cuanto a una pérdida de la salud de las abejas, se manifiesta también en el Sur. Ese interrogante mostró al sector empresarial y al Estado argentino alineados en cuanto a su negativa, y al ámbito de los apicultores con la posición contraria. Curiosamente, una segunda derivación se observó en estos actores, al interrogarse si son los neonicotinoides los causales de esta supuesta crisis. Y aquí es que han entrado a "jugar" fuertemente la comunidad de científicos, en particular los profesionales de la Toxicología, abordando la problemática mediante estudios de diferente tipo. Pero más allá de las coincidencias circunstanciales, es interesante reconocer las similitudes de las hermenéuticas que subyacen a algunos de los actores y las distancias con otros. Allí, puede verse que en lo referido al caso de la salud de las abejas las hermenéuticas que subyacen a SENASA y Bayer mostraron importantes similitudes. 
A su vez, hay otros aspectos involucrados en esta problemática. Por ejemplo, ¿Cómo debe analizarse un paper sobre los efectos de los neonicotinoides financiados por una empresa que los produce? Y del mismo modo, ¿qué hacer frente a la multiplicidad de determinaciones de la pregunta por la salud de las abejas? Indagar los estudios sobre abejas individuales o sobre colmenas, por mencionar una de las líneas de quiebres más notables, dista de ser un único problema con dos aspectos fácilmente complementables. En este sentido, el modo de abordar el problema de la Toxicología y de la Biología del Comportamiento muestra importantes discontinuidades. La primera explicación es de sumo interés: mientras las Toxicología estudia la interacción desde el químico, el otro lo hace desde la abeja. Es la misma interacción pero con dos aproximaciones diferentes. Entonces, ¿es "la misma interacción"?

En esta instancia, cabe reconocer un aspecto que surge de la comparación entre el discurso científico y el que no lo es. El discurso científico generado en la Modernidad tomó la noción de verdad como certeza y con ella la exclusión de otros abordajes potencialmente contradictorios. En las palabras de Kusch ([1975] 2008). Las formas en que se contraponen el "esto es x" propio del discurso académico, enfrentado con un saber popular que muestra la forma "yo creo x". Dice Kusch: "La vinculación que se suele hacer entre pensamiento popular y opinión resulta por demás sospechosa. Proviene en parte de identificar a lo popular con algo distendido y lábil, a lo cual solo podría corresponder la opinión, considerada ésta como un juicio igualmente difuso e indefinido" ([1975] 2008, p. 19).

El asunto es que en términos de políticas públicas, la forma en que se toma esta negación es clave en relación con la existencia: "Decir "así es" no implica solo pasividad ante el mundo, sino que es ante todo una toma de conciencia del darse de uno mismo y de todas las cosas en el conjunto vacío y, por consiguiente, todo lo real, las cosas mismas, dejan de ser cosas para transformarse en símbolos." (Kusch, 2008, p. 35) Y luego: "En ciencia mi existencia tiende a ser víctima de ella, porque lo científico es una manera de colocar en firme la afirmación para poder residualizar lo negativo, aun cuando se trate de mi propia vida." (Kusch, 2008, p. 111). La oposición entre saber y vivir toma forma, pero también en la ausencia de posibilidad de negación se evidencia una ontología que se presenta monista, lejos de cualquier diálogo pretendidamente simétrico.

Considerados en su conjunto, puede verse que la diversidad entre posiciones y hermenéuticas no es reconocida en la toma de decisiones. Estos procesos de simplificación involucran tanto aspectos epistémicos, como metodológicos, ontológicos, como éticos-políticos. El supuesto monista involucra no sólo el privilegio del campo profesional, sino también la prioridad de algunos dominios por sobre otros, como así también la omisión de los resultados que no responden a lo esperado bajo la idea de dificultades en su extrapolación. También el reemplazo del principio de precaución 
por un principio que compara potenciales beneficios y riesgos, es otro de los aspectos que impiden recuperar las complejidades y diversidades del caso.

Procesos de simplificación, omisión y negación son fundamentales para dar cuenta del escenario analizado. Este esquema asumió elementos asociados, tanto la imagen de cierto científico como detector privilegiado de los problemas (y por ende descartando también aquellos que no lo son), como de empresas y Estados que juegan su propio telos. En todos los casos, las políticas públicas en el esquema neoliberal refuerzan la dependencia en la consideración del experto, en políticas que privilegian la producción y ganancia monetaria, por sobre el reconocimiento y prevención de riesgos sociales y ambientales.

\section{Agradecimientos}

Se agradece muy especialmente a la Dra. Paula Blois y a la Dra. Ana Carolina Hecht por su colaboración en el proceso de elaboración del presente artículo.

\section{Referencias}

Agrovoz. (13 de octubre2017). Para quedarse con Monsanto, Bayer le vende a Basf sus semillas y herbicidas en U\$S 6.900 millones. Recuperado de http://agrovoz. lavoz.com.ar/agricultura/para-quedarse-con-monsanto-bayer-le-vende-basf-sussemillas-y-herbicidas-en-us-6900-mill.

Aizen, Marcelo y Harder Lawrence (2009). The global stock of domesticated honey bees is growing slower than agricultural demand for pollination. Current Biology, 19: 915-918.

Aizen, Marcelo, Lucas Garibaldi, Saúl Cunningham y Alexandra Klein (2008). Longterm global trends in crop yield and production reveal no current pollination shortage but increasing pollinator dependency. Current Biology, 18: 1572-1575.

Balbuena María Sol, Léa Tison, Marie-Luise Hahn, Uwe Greggers, Randolf Menzel y Walter Farina (2015). Effects of sublethal doses of glyphosate on honeybee navigation. The Journal of Experimental Biology, 218, 2799-2805. doi:10.1242/jeb.117291.

Bateson Patrick (2012). Behavioural Biology: The Past and a Future. Ethology, 118: 216-221.

Bateson Patrick y Kevin Laland (2013). Tinbergen's four questions: an appreciation and an update. Trends in Ecology E Evolution, 28(12): 712-718.

Bayer (2019). Let's talk about Glyphosate. Recuperado de https://www.bayer.com/en/ glyphosate-roundup.aspx.

Bayer-Beecare (2019). Bee myth. Recuperado de https://beecare.bayer.com/bilder/ upload/dynamicContentFull/Publications/Bee_Myth-Separating_Facts_from_ Fictionk0wgjgwa.pdf. 
Blacquière Tjeerd, Guy Smagghe, Cornelis van Gestel y Veerle Mommaerts (2012). Neonicotinoids in bees: a review on concentrations, side-effects and risk assessment. Ecotoxicology, 21(4): 973-992.

Böschen Stefan, Karen Kastenhofer, Ina Rust y Jens Soentgen (2010). Scientific Nonknowledge and Its Political Dynamics: The Cases of Agri-Biotechnology and Mobile Phoning. Science, Technology \& Human Values, 35(6): 783-811.

Brown Wendy (2017). El pueblo sin atributos. La secreta revolución del neoliberalismo. Barcelona: Malpaso.

CBGnetwork.org (2019). Información Bayer. Recuperado de http://www.cbgnetwork. org/4179.html.

EFSA (28 de febrero 2018). QEA: Conclusions on neonicotinoids 2018. Recuperado de https://www.efsa.europa.eu/sites/default/files/news/180228-QA-Neonics.pdf.

El País (28 de febrero2018). Los insecticidas neonicotinoides son un riesgo para las abejas, según la EFSA. Recuperado de https://elpais.com/elpais/2018/02/28/ciencia/1519817690_532532.html.

Fairbrother Anne, John Purdy, Troy Anderson y Richard Fellk (2014). Risks of Neonicotinoid Insecticides to Honeybees. Environmental Toxicology and Chemistry, 33 (4): 719-731.

Fischer Johannes, Teresa Müller, Anne-Kathrin Spatz, Uwe Greggers, Bernd Grünewald, Randolf Menzel y Dhruba Naug (2014). Neonicotinoids Interfere with Specific Components of Navigation in Honeybees. PLoS ONE, 9(3) e91364.

Frickel, Scott (2004). Chemical Consequences: Environmental Mutagens, Scientist Activism, and the Rise of Genetic Toxicology. New Brunswick, NJ: Rutgers University Press.

Goñalons Carolina y Walter Farina (2018). Impaired associative learning after chronic exposure to pesticides in young adult honey bees. Journal of Experimental Biology, 221,. doi:10.1242/jeb.176644.

Goñalons Carolina y Walter Farina (2015). Effects of Sublethal Doses of Imidacloprid on Young Adult Honeybee Behaviour. PLoS ONE, 10(10): e0140814. doi:10.1371/ journal.pone.0140814.

Herbert Lucila, Diego Vázquez, Andrés Arenas y Walter Farina (2014). Effects of field-realistic doses of glyphosate on honeybee appetitive behaviour. The Journal of Experimental Biology, 217: 3457-3464.

Husserl, Edmund (1991 [1936]). La crisis de las ciencias europeas y la fenomenología trascendental. Barcelona: Editorial Crítica. 
Illich, Ivan (2015 [2006]). Obras reunidas I. México: Fondo de Cultura Económica.

Infocampo.com.ar, (06de abril2018). Cruce entre Etchevehere y apícolas por la muerte de abejas: "El modelo de agroquímicos no va a cambiar". Recuperado de https:// www.infocampo.com.ar/cruce-entre-etchevehere-y-apicolas-por-la-muerte-deabejas-el-modelo-de-agroquimicos-no-va-a-cambiar/.

INTA (2015). Principales características de los insecticidas utilizados en el cultivo de soja. Recuperado de https://inta.gob.ar/sites/default/files/script-tmp-inta-_insecticidas_utilizados_en_soja-_caractersticas.pdf.

Iwasa Takao, Naoki Motoyama, John Ambrose y Michael Roe (2004). Mechanism for the differential toxicity of neonicotinoid insecticides in the honey bee, Apis mellifera. Crop Protection, 23:371-378.

Johnson Reed, Henry Pollock y May Berenbaum (2009). Synergistic interactions between in-hive miticides in Apis mellifera. J Econ Entomol, 102 (2): 474-479.

KleffmannGroup (2012). Mercado Argentino de Productos Fitosanitarios 2012. Recuperado de https://www.casafe.org/pdf/estadisticas/Informe\%20Mercado\%20 Fitosanitario\%202012.pdf.

Daniel Lee Kleinman y Suryanarayanan Sainath (2013). Dying Bees and theSocial Production of Ignorance. Science, Technology, \& Human Values, 38(4) 492-517.

Kusch, Rodolfo (2008 [1975]). La negación en el pensamiento popular. Buenos Aires: Cimarrón.

La Nación. (8 de junio del 2018). Córdoba: confirman que la muerte de 70 millones de abejas fue por un pesticida. https://www.lanacion.com.ar/sociedad/cordoba-confirman-que-la-muerte-de-70-millones-de-abejas-fue-por-un-pesticidanid2142104.

Lundin Ola, Maj Rundlöf, Henrik Smith, Ingemar Fries y Riccardo Bommarco (2015). Neonicotinoid Insecticides and Their Impacts on Bees: A Systematic Review of Research Approaches and Identification of Knowledge Gaps. PLoS ONE, 10(8): e0136928. doi:10.1371/journal.pone.0136928.

Newman Michael y Michael Unger (2003). Fundamentals of Ecotoxicology. 2nd ed. Portland, OR: Lewis Publishers.

Pallitto, Nahuel (2018). Representar e intervenir el comportamiento humano. Un análisis desde la filosofía de la biología (Tesis presentada para optar al título de Doctor) Universidad de Buenos Aires en el área de Filosofía.

Pilling, Edward D y Paul Jepson (1993). Synergism between EBI fungicides and a pyrethroid insecticide in the honeybee (Apis mellifera). Pest Management Science, 39 (4): 293-297. 
Portal Apícola (28 de octubre2013). Comunicado. SENASA y su visión sobre los neonicotinoides. Recuperado de http://www.apicultura.entupc.com/nuestrarevista/ nueva/notas/28-10-13-Principal.html.

Ricoeur, Paul (1975). "Existencia y Hermenéutica" en Hermenéutica y estructuralismo. Buenos Aires: Ediciones Megápolis.

SADA. Sociedad Argentina de Apicultores (2019). Convertidor en protector. https:// sada.org.ar/protegeunacolmena/.

Science (29 de junio de 2017). Controversial pesticides can decimate honey bees, large study finds. Recuperado de https://www.sciencemag.org/news/2017/06/controversial-pesticides-can-decimate-honey-bees-large-study-finds.

Stagnaro, Adriana (2006). De antropóloga externa a antropóloga local. Diferentes modos de implicación. Cuadernos de Antropología Social. 23, 81-103.

Stanford. (2019). Hermenéutica. Recuperado de https://plato.stanford.edu/entries/ hermeneutics/.

Suchail Séverine, David Guez y Luc Belzunces (2001). Discrepancy between acute and chronic toxicity induced by imidacloprid and its metabolites in Apis mellifera. National Library of Medicine, 20(11): 2482-6.

Tan Ken, Weiwen Chen, Shihao Dong, Xiwen Liu, Yuchong Wang y James Nieh (2015). A neonicotinoid impairs olfactory learning in Asian honey bees (Apis cerana) exposed as larvae or as adults. Sci Rep, 18(5): 1-8.

Thompson Helen y Selwyn Wilkins (2003). Assessment of the synergy and repellency of pyrethroid/fungicide mixtures. Boletín de insectologia, 56 (1): 131-134.

Tosi Simone, Giovanni Burgio y James Nieh (2017). A common neonicotinoid pesticide, thiamethoxam, impairs honey bee flight ability. Scientific Reports, 7.

Tsvetkov Nadejda, Samson-Robert Olivier, Keshna Sood, Harshillkumar Patel, Daniel Malena, Pratik Gajiwala, Philip Maciukiewicz, Valerie Fournier y Amro Zayed (2017). Chronic exposure to neonicotinoids reduces honey bee health near corn crops. Science, 30, 356 (6345): 1395-1397.

vanEngelsdorp Dennis, Jay Evans, Claude Saegerman, Chris Mullin, Eric Haubruge, Bach Kim Nguyen, Maryann Frazier, Jim Frazier, Diana Cox-Foster, Yanping Chen, Robyn Underwood, David Tarpy y Jeffery Pettis (2009). Colony Collapse Disorder: A Descriptive Study. PLoS ONE, 4: e6481.

Vázquez Diego, Natalia Ilina, Eduardo Pagano, Jorge Zavala y Walter Farina (2018). Glyphosate affects the larval development of honey bees depending on the susceptibility of colonies. PLoS ONE, 13(10): e0205074.

Walton, Roberto (1993). Husserl, mundo, conciencia y temporalidad. Buenos Aires: Editorial Almagesto. 
Whitehorn Penelope R, Stephanie O'Connor, Felix Wackers y Dave Goulson (2012). Neonicotinoid Pesticide Reduces Bumble Bee Colony Growth and Queen Production. Science, 20, 336 (6079): 351-352.

Williamson Sally M, Sarah Willis y Geraldine Wright (2014). Exposure to neonicotinoids influences the motor function of adult worker honeybees. Ecotoxicology, 23(8): 1409-1418.

Woodcock Ben A, James Bullock, Richard Shore, Matthew Heard, Gloria Pereira, Redhead, Ridding Lucy, Dean Hannah, Sleep Darren, Henrys P, Peyton J, Sarah Hulmes, Lucy Hulmes, Miklos Sárospataki, Christoph Saure y Edwards Pywell (2017). Country-specific effects of neonicotinoid pesticides on honey bees and wild bees. Science, 30; 356 (6345):1393-1395

Wright David y Pamela Welbourn (2002). Environmental Toxicology. Cambridge: Cambridge University Press.

Wynne, Brian (2004). May the sheep safely graze? A reflexive view of the expert-lay knowledge divide. Risk, environment and modernity: towards a new ecology. Lash, S., Szerszynski, B. y Wynne, B. (eds.). Sage.

Zhu Yu-Cheng, Jianxiu Yao, John Adamczyk y Randall Luttrell (2017). Synergistic toxicity and physiological impact of imidacloprid alone and binary mixtures with seven representative pesticides on honey bee (Apis mellifera). PLoS One, 3;12(5):e0176837.

\section{Sobre el autor}

Guillermo Folguera es Dr. y Profesor de la Universidad de Buenos Aires e Investigador en CONICET. Coordina el Grupo de Filosofía de la Biología (UBA-CONICET). Correo Electrónico: guillefolguera@yahoo.com.ar. https://orcid.org/0000-00024990-7039 


\title{
CUHSO
}

Fundada en 1984, la revista CUHSO es una de las publicaciones periódicas más antiguas en ciencias sociales y humanidades del sur de Chile. Con una periodicidad semestral, recibe todo el año trabajos inéditos de las distintas disciplinas de las ciencias sociales y las humanidades especializadas en el estudio y comprensión de la diversidad sociocultural, especialmente de las sociedades latinoamericanas y sus tensiones producto de la herencia colonial, la modernidad y la globalización. En este sentido, la revista valora tanto el rigor como la pluralidad teórica, epistemológica y metodológica de los trabajos.

\author{
EDITOR \\ Matthias Gloël \\ COORDINADORA EDITORIAL \\ Claudia Campos Letelier \\ CORRECTOR DE ESTILO Y DISEÑAdOR \\ Ediciones Silsag \\ TRADUCTOR, CORRECTOR LENGUA INGLESA \\ Aurora Sambolin Santiago \\ SITIO WEB \\ cuhso.uct.cl \\ E-MAIL \\ cuhso@uct.cl \\ LICENCIA DE ESTE ARTÍCULO \\ Creative Commons Atribución Compartir Igual 4.0 Internacional
}

